REVISTA DE PSIQUIATRIA INFANTO-JUVENIL

ISSN 1130-9512 | E-ISSN 2660-7271

Volúmen 37, número 4, octubre-diciembre de 2020, pp. 54-55

Este artículo está bajo licencia Creative Commons

BY-NC-ND 4.0

DOI: $10.31766 /$ revpsij.v37n4a5

\title{
Comentario de libro
}

\section{Susana Cruylles ${ }^{1}$ \\ Prudencio Rodríguez Ramos ${ }^{2}$}

1. Psicóloga Clínica, Terapeuta de Familia, España.

2. Psiquiatra sin especialidad oficialmente reconocida en la infancia y la adolescencia, España.
El estrés postraumático en niños $y$ adolescentes. Comentarios de cuatro libros publicados en los últimos años por el Dr. Victor G. Carrión
El tratamiento del estrés postraumático en niños y adolescentes, dada su complejidad en causalidad, evaluación diagnóstica, manifestaciones clínicas, evolución y tratamiento, requiere una precisa y amplia formación especializada para su diagnóstico y tratamiento. Hay cuatro libros publicados en los últimos años (2016-2019) por el Dr. Víctor G. Carrión que, juntos, contribuyen a tan específica formación y muestran la enorme importante aportación que este profesor está haciendo en la psiquiatría infantil y en el tratamiento de dicha patología. El Dr. Carrión es el John A. Turner M.D. Endowed Chair of Child and Adolescent Psychiatry en la Universidad de Stanford, California, donde dirige un programa de estrés infantojuvenil: The Early Life Stress and Resilience Program

(http://med.stanford.edu/elspap.html).

Aunque estos libros (no descritos por orden de publicación), están dirigidos al estrés postraumático en niños y adolescentes, abordan muchos aspectos de la psiquiatría infantil.

El primero que se describe a continuación, Assessing and Treating Youth exposed to Traumatic Stress, APA Publishing 2019 (1), es un manual-guía general en el que el Dr. Carrión es el editor. Participan autores, expertos en la materia: profesores, investigadores y profesionales clínicos. Se describe cómo hacer una evaluación clínica de un niño o adolescente con estrés postraumático, con toda su diversidad, comorbilidad y complejidad sintomatológica. Además, el libro presenta diferentes tratamientos terapéuticos generales y específicos al respecto, que hay en la actualidad, como Cue Centered Therapy, Trauma -Focus Cognitive Behavioral Therapy o Child -Parent interventions. El libro presenta, en un tercer apartado, diferentes problemas clínicos asociados, como la disociación, dificultades en el sueño, suicidio y abuso de sustancias. En la última parte del libro, se describen sistemas integrados de cuidados para el tratamiento de estrés postraumático.

El segundo de los libros que se reseña es Terapia de Claves traumáticas: Manual de intervención para niños y adolescentes con sintomas postraumáticos, Gedisa 2019 (traducido del original: Cue-Centered Therapy for youth experiencing posttraumatic symptoms, Oxford University Press 2016) (2). Es un manual de tratamiento del estrés postraumático, sesión a sesión, creado por el Dr. Carrión. Todo el tratamiento, detalladamente descrito, está basado en la neurociencia del estrés postraumático y aborda la variada sintomatología postraumática, que se suele manifestar en los distintos ámbitos: conductual, cognitivo, emocional y físico. Describe las claves traumáticas y como éstas causan la sintomatología observada. La finalidad de este método de tratamiento, híbrido de varias terapias eficaces, es fortalecer al niño y adolescente a través de la psicoeducación, de la revisión de su narrativa biográfica y de la exposición a las claves traumáticas.

De aquí pasamos al siguiente libro, maravilloso en su sencillez para hablar de algo tan complejo y pionero, como la neurociencia del estrés postraumático pediátrico: Neuroscience of Pediatric PTSD, Oxford University Press 2017, escrito junto con el Dr. Carl Weems (3). Describe todas las investigaciones 
REVISTA DE PSIQUIATRIA INFANTO-JUVENIL ISSN 1130-9512 | E-ISSN 2660-7271

Volúmen 37, número 4, octubre-diciembre de 2020, pp. 54-55

Este artículo está bajo licencia Creative Commons

BY-NC-ND 4.0

que hasta 2017 existen en la literatura sobre el impacto del estrés traumático en el cerebro del niño y su funcionamiento: en las funciones ejecutivas, la memoria, procesamiento emocional, disociación, sueño y conductas autolíticas. Hay además un capítulo sobre los estudios de comorbilidad en el Trastorno por Estrés Post-traumático (TEPT) pediátrico, otro sobre los resultados de tratamiento y por último indica futuras direcciones a tomar en este campo de investigación. Todos los capítulos presentan el mismo formato: describen la anatomía del cerebro a la que hacen mención, los estudios realizados en animales y en adultos, para llegar a los realizados en la población infantojuvenil.

Hasta aquí todo lo enfocado a evaluación, tratamiento y neurociencia del estrés postraumático en población pediátrica. Vamos un paso más, con el proyecto del Dr. Carrión: hacer prevención y tratamiento integral del estrés traumático.

El último libro descrito es un manual práctico que describe la utilidad, beneficios (y necesidad) de la práctica de mindfulness para salud mental de la población infantojuvenil: Applied Mindfulness: Approaches in Mental Health for Children and Adolescentes, APA Publishing 2019 (4). Esta editado junto con el Dr. John Rettger, quien fue director de mindfulness del departamento de Early Life Stress and Resilience Program de la Universidad de Stanford. Participan varios autores expertos en la materia. Empieza describiendo la aplicación de mindfulness en la población clínica infantojuvenil: ansiedad, depresión, trastorno por déficit de atención con hiperactividad, duelos, abuso de sustancias y autismo. En la segunda parte del libro, se acerca a la práctica de mindfulness en poblaciones vulnerables: juventud inmigrante y penales. Y por último propone varios entornos para la práctica de mindfulness con niños y adolecentes, como el domicilio, la escuela, o la naturaleza.

Quien profundice en estos libros podrá empezar a comprender la complejidad del estrés postraumático en la infancia, en diagnóstico y tratamiento, su gravedad, y las consecuencias a medio y largo plazo para el desarrollo y el bienestar de quienes lo sufren.
El estrés postraumático en niños y adolescentes. Comentarios de cuatro libros publicados en los últimos años del Dr. Víctor $G$. Carrión.

Y por ello, la necesidad de formación específica al respecto.

Por último, una sugerencia: que se realicen traducciones de los tres libros descritos no traducidos, para hacerlos accesibles y formar a profesionales que no tienen la oportunidad de ser angloparlantes.

\section{REFERENCIAS}

1. Carrión VG. Assessing and Treating Youth exposed to Traumatic Stress. APA Publishing; 2019. 528 p. ISBN-10: 1615371427, ISBN-13: 978-1615371426.

2. Carrión VG. Cue-Centered Therapy for Youth Experiencing Posttraumatic Symptoms. Cue-Centered Therapy for Youth Experiencing Posttraumatic Symptoms. Oxford University Press; 2016. 224 p. ISBN-10: 0190201320, ISBN-13: 978-0190201326 / Terapia de Claves traumáticas: Manual de intervención para niños y adolescentes con síntomas postraumáticos; Gedisa 2019. 224 p. ISBN: 978-84-17341-93-0

3. Carrión VG \& Weems C. Neuroscience of Pediatric PTSD. Oxford University Press; 2017. 200 p. ISBN-13: 978-0190201968.

4. Carrión VG \& Rettger J. Applied Mindfulness: Approaches in Mental Health for Children and Adolescents. APA Publishing; 2019. 394 p. ISBN10:1615372121, ISBN-13 :978-1615372126. 